J. Korean Math. Soc. 42 (2005), No. 1, pp. 101110

\title{
REMARKS ON THE KKM PROPERTY FOR OPEN-VALUED MULTIMAPS ON GENERALIZED CONVEX SPACES
}

\author{
HoOnJOo KIM* AND SEHIE PARK
}

\begin{abstract}
Abstrac't. Let $(X, D ; \Gamma)$ be a $G$-convex space and $Y$ a Hausdorff space. Then $\mathfrak{A}_{c}^{\kappa}(X, Y) \subset \mathfrak{K O}(X, Y)$, where $\mathfrak{A}_{c}^{\kappa}$ is an admissible class (due to Park) and $\mathfrak{K O}$ denotes the class of multimaps having the KKM property for open-valued multimaps. This new result is used to obtain a KKM type theorem, matching theorems, a fixed point theorem, and a coincidence theorem.
\end{abstract}

\section{Introduction}

The KKM theory of generalized convex spaces (or $G$ - convex spaces) has been developed mainly by the second author and followed by a number of other authors; for the literature, see the references at the end of the present paper.

In this paper, our main aims are to improve one of our earlier results [11, Theorem 11] and to obtain some of its applications. In fact, let $(X, D ; \Gamma)$ be a $G$-convex space, $Y$ a Hausdorff space, and $G: D \multimap Y$ an open-valued multimap. If an admissible map $F \in \mathfrak{A}_{c}^{\kappa}(X, Y)$ (due to Park [2-4]) satisfies $F\left(\Gamma_{A}\right) \subset G(A)$ for all finite subset $A$ of $D$, then the family $\{G(z)\}_{z \in D}$ of values of $G$ has the finite intersection property. In [11], this was proved under the restriction that $Y$ is $T_{1}$ and regular.

Section 2 deals with preliminaries taken from a recent work of the second author [9]. In Section 3, we prove our main result and apply it to obtain a generalized form of a KKM theorem, matching theorems for closed [resp. open] valued multimaps, a fixed point theorem, and a coincidence theorem.

Received August 11, 2003.

2000 Mathematics Subject Classification: 47H10, 54H25, 55M20.

Key words and phrases: KKM principle, generalized convex ( $G$-convex) spaces, I-convex subsets, multimaps having the KKM property.

*This work was supported by grant No. KMS 2001-02 from the KOSEF. 


\section{The KKM theorem for $G$-convex spaces}

A multimap (or simply, a map) $F: X \multimap Y$ is a function from a set $X$ into the power set of $Y$; that is, a function with the values $F(x) \subset Y$ for $x \in X$ and the fibers $F^{-}(y):=\{x \in X \mid y \in F(x)\}$ for $y \in Y$. For $A \subset X$, let $F(A):=\bigcup\{F(x) \mid x \in A\}$. Throughout this paper, we assume that multimaps have nonempty values otherwise explicitly stated or obvious from the context.

For topological spaces $X$ and $Y$, a multimap $F: X \multimap Y$ is said to be upper semicontinuous (u.s.c.) [resp. lower semicontinuous (l.s.c.)] if for each closed [resp. open] set $B \subset Y, F^{-}(B):=\{x \in X \mid F(x) \cap B \neq \emptyset\}$ is closed [resp. open] in $X$.

Let $\langle D\rangle$ denote the set of all nonempty finite subsets of a set $D$.

A generalized convex space or a $G$-convex space $(X, D ; \Gamma)$ consists of a topological space $X$, a nonempty set $D$, and a multimap $\Gamma:\langle D\rangle \multimap X$ such that for each $A \in\langle D\rangle$ with its cardinal $|A|=n+1$, there exists a continuous function $\phi_{A}: \Delta_{n} \rightarrow \Gamma_{A}:=\Gamma(A)$ such that $J \in\langle A\rangle$ implies $\phi_{A}\left(\Delta_{J}\right) \subset \Gamma_{J}:=\Gamma(J)$. In certain cases, we may assurne $\phi_{A}\left(\Delta_{n}\right)=\Gamma_{A}$.

Note that $\Delta_{n}$ is an $n$-simplex with vertices $v_{0}, v_{1}, \ldots, v_{n}$, and $\Delta_{J}$ the face of $\Delta_{n}$ corresponding to $J \in\langle A\rangle$; that is, if $A=\left\{a_{0}, a_{1}, \ldots, a_{n}\right\}$ and $J=\left\{a_{i_{0}}, a_{i_{1}}, \ldots, a_{i_{k}}\right\} \subset A$, then $\Delta_{J}=\operatorname{co}\left\{v_{i_{0}}, v_{i_{1}}, \ldots, v_{i_{k}}\right\}$.

In case to emphasize $X \supset D,(X, D ; \Gamma)$ will be denoted by $(X \supset D ; \Gamma)$.

For a $G$-convex space $(X \supset D ; \Gamma)$, a subset $Y \subset X$ is said to be $\Gamma$-convex if for each $N \in\langle D\rangle, N \subset Y$ implies $\Gamma_{N} \subset Y$.

Examples of $G$-convex spaces can be found in $[5,6,8]$ and references therein.

For a $G$-convex space $(X, D ; \Gamma)$, a multimap $F: D \multimap X$ is called a $K K M$ map if

$$
\Gamma_{N} \subset F(N) \quad \text { for each } N \in\langle D\rangle \text {. }
$$

The following is a KKM theorem for $G$-convex spaces $[5,6]$ :

Theorem 2.1. Let $(X, D ; \Gamma)$ be a $G$-convex space and $F: D \multimap X$ a map such that

(1) $F$ has closed [resp. open] values; and

(2) $F$ is a KKM map.

Then $\{F(z)\}_{z \in D}$ has the finite intersection property.

Further, if

(3) $\bigcap_{z \in M} \overline{F(z)}$ is compact for some $M \in\langle D\rangle$, 
then we have

$$
\bigcap_{z \in I} \overline{F(z)} \neq \emptyset .
$$

REMARK. There have appeared several variations of Theorem 2.1; sec $[6,12]$.

Let $(X, D ; \Gamma)$ be a $G$-convex space and $Y$ a topological space. A multimap $F^{\prime}: X \multimap Y$ is said to have the KKM property if, for any map $G: D \multimap Y$ with closed [resp. open] values satisfying

$$
F\left(\Gamma_{A}\right) \subset G(A) \quad \text { for all } A \in\langle D\rangle .
$$

the family $\{G(z)\}_{z \in I}$ lias the finite intersection property. We denote

$$
\mathfrak{K}(X, Y):=\{F: X \multimap Y \mid F \text { has the KKM property }\} \text {. }
$$

Some authors use the notation $K K M(X, Y)$. Note that $1_{X} \in \mathfrak{K}(X, X)$ by Theorem 2.1. Moreover, if $F: X \rightarrow Y$ is a continuous single-valued map or if $F: X \multimap Y$ has a contimuous selection, then it is easy to check that $F \in \mathfrak{K}(X . Y)$. Note that there are many known selection theorems due to Michael and others.

From now on. $\mathfrak{K C}$ denote $S$ the class $\mathfrak{K}$ for closed-valued maps $G$, and $\mathfrak{K O}$ for opeu-valued maps $G$.

From Theorem 2.1, we derived the following basic coincidence theorox in [7]:

Theorex 2.2. Let $(X, D ; \Gamma)$ be a $G$-convex space. $Y$ a topological space, $S: D \multimap Y, T: X \multimap Y$, and $F \in \mathfrak{R C}(X, Y)$. Suppose that

(1) $S$ has open values;

(2) for ach $y \in F(X)$. $M \in \in\left\langle S^{-}(y)\right\rangle$ implies $\Gamma_{M} \subset T^{-}(y)$; and

(3) $\overline{F(X)} \subset S(N)$ for some $N \in\langle D\rangle$.

The'I $F$ and $T$ have a coincidence point $x_{*} \in X$; that is, $F\left(x_{*}\right) \cap T\left(x_{*}\right) \neq$ $\emptyset$.

Throrem 2.2 is applied in [7] to the Fan-Browder theorem, $\Phi$-spaces, and $\omega$-connected spaces.

Similarly, for the class $\mathfrak{K O}(X, Y)$, we have the following basic coincidence theorem in $[9]$ :

THeORen $2.2^{\prime}$. Let $(X, D ; \Gamma)$ be a $G$-convex space, $Y$ a topological space. $S: D \multimap Y . T: X \multimap Y$, and $F \in \mathfrak{K O}(X, Y)$. Suppose that

(1) S has closed values:

(2) for each $y \in F(X), M \in\left\langle S^{-}(y)\right\rangle$ implies $\Gamma_{M} \subset T^{-}(y)$; and

(3) $Y=S(N)$ for some $N \in\langle D\rangle$. 
Then $F$ and $T$ have a coincidence point $x_{*} \in X$; that is, $F\left(x_{*}\right) \cap T\left(x_{*}\right) \neq$ $\emptyset$.

REMARK. It would be possible to replace the class $\mathfrak{K}$ in this paper by the so-called $S$-KKM class introduced by some authors. However, we will not do this in the present paper.

By putting $X=Y$ and $F=1_{X}$ in Theorems 2.2 and $2.2^{\prime}$, we have a general form of the Fan-Browder theorem for $G$-convex spaces:

Theorem 2.3. Let $(X, D ; \Gamma)$ be a $G$-convex space, and $S: D \multimap X$, $T: X \multimap X$ two maps satisfying

(1) for each $z \in D, S(z)$ is open [resp. closed];

(2) for each $y \in X, M \in\left\langle S^{-}(y)\right\rangle$ implies $\Gamma_{M} \subset T^{-}(y)$; and

(3) $X=S(N)$ for some $N \in\langle D\rangle$.

Then $T$ has a fixed point $x_{0} \in X$; that is, $x_{0} \in T\left(x_{0}\right)$.

Theorem 2.3 is obtained in [7] and applied to various forms of the Fan--Browder theorem, the Ky Fan intersection theorem, and the Nash equilibrium theorem for $G$-convex spaces.

From Theorem 2.3, we deduced the following in [9]:

THEOREM $2.3^{\prime}$. Let $(X \supset D ; \Gamma)$ be a $G$-convex space and $A: X \multimap X$ be a multimap such that $A(x)$ is $\Gamma$-convex for each $x \in X$. If there exist $z_{1}, z_{2} \ldots, z_{n} \in D$ and nonempty open [resp. closed] subsets $G_{i} \subset A^{-}\left(z_{i}\right)$ for $i=1,2, \ldots, n$ such that $X=\bigcup_{i=1}^{n} G_{i}$, then $A$ has a fixed point.

A polytope is a finite dimensional compact convex subset of a t.v.s. Let $X$ and $Y$ be topological spaces. An admissible class $\mathfrak{A}_{c}^{\kappa}(X, Y)$ of maps $T: X \multimap Y$ is one such that, for each compact subset $K$ of $X$, there exists a map $S \in \mathfrak{A}_{c}(K, Y)$ satisfying $S(x) \subset T(x)$ for all $x \in K$; where $\mathfrak{A}_{c}$ is consisting of finite composites of maps in $\mathfrak{A}$, and $\mathfrak{A}$ is a class of maps satisfying the following properties:

(i) $\mathfrak{A}$ contains the class $\mathbb{C}$ of (single-valued) continuous functions;

(ii) each $F \in \mathfrak{A}_{c}$ is upper semicontinuous and compact-valued; and

(iii) for each polytope $P$, each $F \in \mathfrak{A}_{c}(P, P)$ has a fixed point, where the intermediate spaces of composites are suitably chosen for each $\mathfrak{A}$.

Examples of $\mathfrak{A}$ are continuous functions $\mathbb{C}$, the Kakutani maps $\mathbb{K}$ (with convex values and codomains are convex spaces), the Aronszajn maps $\mathbb{M}$ (with $R_{\delta}$ values), the acyclic maps $\mathbb{V}$ (with acyclic values), the Powers maps $\mathbb{V}_{c}$, the O'Neil maps $\mathbb{N}$ (continuous with values of one 
or $m$ acyclic components, where $m$ is fixed), the approachable maps $\mathbb{A}$ (whose domains and codomains are subsets of uniform spaces), admissible maps of Góniewicz, $\sigma$-selectional maps of Haddad and Lasry, permissible maps of Dzedzej. and others. Further, $\mathbb{K}_{c}^{+}$due to Lassonde, $\mathbb{V}_{c}^{+}$ due to Park et al.. and approximable maps $\mathbb{A}^{\kappa}$ due to Ben-El-Mechaiekh and Idzik are examples of $\mathfrak{A}_{c}^{\kappa}$. For the literature, see [2-4]. Many other careless authors mistook $\mathfrak{A}$ for $\mathcal{U}$.

Lemma 2.4. Let $(X, D ; \Gamma)$ be a $G$-convex space and $Y$ a Hausdorff space. Then $\mathfrak{A}_{c}^{\kappa}(X, Y) \subset \mathfrak{K C}(X, Y)$.

This is given as [11, Corollary]. In the same paper, we showed that $\mathfrak{A}_{c}^{\kappa}(X, Y) \subset \mathfrak{K O}(X, Y)$ whenever $Y$ is $T_{1}$ regular [11, Theorem 11] in view of the following [11, Lemma]:

Lemia 2.5. Let $(X . D ; \Gamma)$ be a $G$-convex space, $|D|=n+1, Y$ a regular space, and $F: X \multimap Y$ a compact-valued u.s.c. map. If $G: D \multimap Y$ is an open-valued map such that

(1) for each $J \in\langle D\rangle, F\left(\Gamma_{J}\right) \subset G(J)\left[\right.$ or $\left.F\left(\phi_{D}\left(\Delta_{J}\right)\right) \subset G(J)\right]$;

then there is a closed-valued map $H: D \multimap Y$ such that $H(x) \subset G(x)$ for all $x \in D$; and

(2) $F\left(\phi_{D}\left(\Delta_{J}\right)\right) \subset H(J)$ for each $J \subset D$;

where $\Delta_{J}$ is the face of $\Delta_{n}$ corresponding to $J$ and $\phi_{D}: \Delta_{n} \rightarrow \Gamma_{D}$ a continuous map such that $\phi_{D}\left(\Delta_{J}\right) \subset \Gamma_{J}$.

In [11], it was assumed thoroughly that $D \subset X$, which is now redundant in our present definition of $(X, D ; \Gamma)$.

\section{Main results}

Now we show that regularity of $Y$ in [11, Theorem 11] can be eliminated, or that $\mathfrak{K C}$ can be replaced by $\mathfrak{K O}$ in Lemma 2.4, as follows:

ThEOREM 3.1. Let $(X, D ; \Gamma)$ be a $G$-convex space and $Y$ a Hausdorff space. Then $\mathfrak{A}_{c}^{\kappa}(X . Y) \subset \mathfrak{K O}(X, Y)$.

Proof. Let $F \in \mathfrak{A}_{c}^{\kappa}(X, Y)$ and $G: D \multimap Y$ an open-valued multimap satisfying $F\left(\Gamma_{A}\right) \subset G(A)$ for all $A \in\langle D\rangle$. Let $A$ have $n+1$ elements. Then $\phi_{A}\left(\Delta_{n}\right) \subset \Gamma_{A}$, where $\phi_{A}: \Delta_{n} \rightarrow \Gamma_{A}$ is a continuous function such that $J \in\langle A\rangle$ implies $\phi_{A}\left(\Delta_{J}\right) \subset \Gamma_{J}$ in the definition of $G$-convex spaces. Since $\phi_{A}\left(\Delta_{n}\right)$ is compact and $F \in \mathfrak{A}_{c}^{\kappa}(X, Y)$, there exists a map $F^{\prime} \in \mathfrak{A}_{c}\left(\phi_{A}\left(\Delta_{n}\right), Y\right)$ such that $F^{\prime}(x) \subset F(x)$ for $x \in \phi_{A}\left(\Delta_{n}\right)$. Note that 
$F^{\prime}\left(\phi_{A}\left(\Delta_{n}\right)\right) \subset F\left(\Gamma_{A}\right) \subset G(A)$. Since $F^{\prime}$ is u.s.c. and compact-valued, $F^{\prime}\left(\phi_{A}\left(\Delta_{n}\right)\right)$ is Hausdorff and compact, hence is normal and regular. Moreover,

$$
F^{\prime}\left(\phi_{A}\left(\Delta_{n}\right)\right) \subset \bigcup_{a \in A} G(a) \cap F^{\prime}\left(\phi_{A}\left(\Delta_{n}\right)\right)=G^{\prime}(A),
$$

where $G^{\prime}: A \multimap F^{\prime}\left(\phi_{A}\left(\Delta_{n}\right)\right)$ is defined by $G^{\prime}(a):=G(a) \cap F^{\prime}\left(\phi_{A}\left(\Delta_{n}\right)\right)$ for all $a \in A$. Note that $G^{\prime}$ has (relatively) open values such that, for each $J \in\langle A\rangle$,

$$
F^{\prime}\left(\phi_{A}\left(\Delta_{J}\right)\right) \subset F\left(\Gamma_{J}\right) \subset G(J) \Rightarrow F^{\prime}\left(\phi_{A}\left(\Delta_{J}\right)\right) \subset G^{\prime}(J) .
$$

Define a map $\Gamma^{\prime}:\langle A\rangle \multimap \phi_{A}\left(\Delta_{n}\right)$ by $\Gamma^{\prime}(J):=\phi_{A}\left(\Delta_{J}\right)$ for each $J \in\langle A\rangle$. Then $\left(\phi_{A}\left(\Delta_{n}\right), A ; \Gamma^{\prime}\right)$ is a $G$-convex space. Therefore, by Lemma 2.5 , there is a closed-valued map $H: A \multimap F^{\prime}\left(\phi_{A}\left(\Delta_{n}\right)\right)$ such that $H(a) \subset$ $G^{\prime}(a)$ for all $a \in A$ and

$$
F^{\prime}\left(\phi_{A}\left(\Delta_{J}\right)\right) \subset H(J) \text { for each } J \in\langle A\rangle .
$$

Note that $F^{\prime} \phi_{A} \in \mathfrak{A}_{c}\left(\Delta_{n}, F^{\prime}\left(\phi_{A}\left(\Delta_{n}\right)\right)\right) \subset \mathfrak{K C}\left(\Delta_{n}, F^{\prime}\left(\phi_{A}\left(\Delta_{n}\right)\right)\right)$ and hence, by the definition of the class $\mathfrak{K C},\{H(a)\}_{a \in A}$ has the finite intersection property. Since

$$
\emptyset \neq \bigcap_{a \in A} H(a) \subset \bigcap_{a \in A} G^{\prime}(a)=\left[\bigcap_{a \in A} G(a)\right] \cap F^{\prime}\left(\phi_{A}\left(\Delta_{n}\right)\right),
$$

we conclude that $\bigcap_{a \in A} G(a) \neq \emptyset$. Since $A \in\langle D\rangle$ is arbitrary, $\{G(z)\}_{z \in D}$ has the finite intersection property. Therefore, we conclude that $F \in$ $\mathfrak{K O}(X, Y)$.

Theorem 3.1 can be restated as follows:

Theorem 3.2. Let $(X, D ; \Gamma)$ be a $G$-convex space, $Y$ a Hausdorff space, $F \in \mathfrak{A}_{c}^{\kappa}(X, Y)$, and $G: D \multimap Y$ such that

(1) for each $x \in D, G(x)$ is open; and

(2) for any $N \in\langle D\rangle, F\left(\Gamma_{N}\right) \subset G(N)$.

Then $\{G(x) \mid x \in D\}$ has the finite intersection property.

Theorem 3.2 improves [11, Theorem 11], where $Y$ is assumed to be $T_{1}$ and regular.

Some of other results in earlier works of the authors mentioned in the end of $[11$, Theorem 11] can be also improved. For example, a KKM type theorem [10, Theorem 6] can be stated as follows: 
Theoren 3.3. Let $(X . D ; \Gamma)$ be a $G$-convex space, $A \in\langle D\rangle, Y$ a topological space, $G: A \multimap Y$ a map, and $F \in \mathfrak{K O}(X, Y)$. Suppose that

(1) for each $x \in A . G(x)$ is open in $Y$; and

(2) for any $N \in\langle A\rangle$. $F\left(\Gamma_{N}\right) \subset G(N)$.

Then $F\left(\Gamma_{A}\right) \cap \bigcap\{G(a) \mid a \in A\} \neq \emptyset$.

Proof. Suppose the conclusion does not hold. Then $F\left(\Gamma_{A}\right) \subset S(A)$ where $S(x)=F\left(\Gamma_{A}\right) \backslash G(x)$ for $x \in A$. Define a map $\Gamma^{\prime}: A \multimap \Gamma_{A}$ by $\Gamma^{\prime}(J):=\Gamma_{J} \cap \Gamma_{A}$ for each $J \in\langle A\rangle$, then $\left(\Gamma_{A}, A ; \Gamma^{\prime}\right)$ is a $G$-convex space. Then conditions (1) and (3) in Theorem $2.2^{\prime}$ are satisfied for $\left(\left(\Gamma_{A} \cdot A ; \Gamma^{\prime}\right)\right.$, $\left.F\left(\Gamma_{A}\right)\right)$ instead of $((X . D ; \Gamma), Y)$. Let $H: F\left(\Gamma_{A}\right) \multimap \Gamma_{A}$ and $T: \Gamma_{A} \multimap$ $F\left(\Gamma_{A}\right)$ be defined by $H(y):=\bigcup\left\{\Gamma_{M}^{\prime} \mid M \in\left\langle S^{-}(y)\right\rangle\right\}$ for $y \in F\left(\Gamma_{A}\right)$ and $T(x):=H^{-}(x)$ for $x \in \Gamma_{A}$. Then (2) in. Theorem $2.2^{\prime}$ is satisfied, hence $T$ and $F$ have a coincidence point $x_{0} \in \Gamma_{A}$; that is. $T\left(x_{0}\right) \cap F\left(x_{0}\right) \neq \emptyset$. For $y \in T\left(x_{0}\right) \cap F\left(x_{0}\right)$, we have $x_{0} \in T^{-}(y)=\bigcup\left\{\Gamma_{M}^{\prime} \mid M \in\left\langle S^{-}(y)\right\rangle\right\}$, and hence there exists a finite set $M \subset S^{-}(y) \subset A$ such that $x_{0} \in \Gamma_{M}^{\prime}$. Since $M \in\left\langle S^{-}(y)\right\rangle$ implies $y \in S(x)$ for all $x \in M$, we have $y \in$ $F\left(x_{0}\right) \cap \bigcap\{S(x) \mid x \in M\}$. Therefore $\emptyset \neq F\left(\Gamma_{M I}^{\prime}\right) \cap \bigcap\{S(x) \mid x \in M\} \subset$ $F\left(\Gamma_{M}\right) \cap \bigcap\{S(x) \mid x \in M\}$; that is, $F\left(\Gamma_{M}\right) \not \subset G(M)$. This contradicts (2). This completes our proof.

The following is a matching theorem:

Theorfar 3.4. Let $(X, D ; \Gamma)$ be a $G$-convex space. $Y$ a topological space, $F \in \mathfrak{K O}(X . Y)$, and $A \in\langle D\rangle$. Let $S: A \multimap Y$ be a map such that

(1) S has closed values; and

(2) $S(A)=Y$.

Then there exists a $B \in\langle A\rangle$ such that $F\left(\Gamma_{B}\right) \cap \bigcap\{S(b) \mid b \in B\} \neq \emptyset$.

Proof. Suppose that the conclusion does not hold. Define a map $G: A \multimap Y$ by $G(a):=Y \backslash S(a)$ for $a \in A$. Then each $G(a)$ is open and for each $B \in\langle A\rangle$.

$$
F\left(\Gamma_{I 3}\right) \subset \bigcup_{b \in B} Y \backslash S(b)=\bigcup_{b \in B} G(b)=G(B) .
$$

Since $F \in \mathfrak{K O}(X . Y)$, the family $\{G(a)\}_{a \in A}$ has the finite intersection property. Therefore $\bigcap\{G(a) \mid a \in A\} \neq \emptyset$ and hence $S(A) \neq Y$, a contradiction. 
REMARK. A particular form of Theorem 3.4 is given by Balaj [1, Lemma 1].

We have another matching theorem:

Theorem $3.4^{\prime}$. Let $(X, D ; \Gamma)$ be a $G$-convex space, $Y$ a topological space, $F \in \mathfrak{K C}(X, Y)$, and $A \in\langle D\rangle$. Let $T: A \multimap Y$ be a map such that

(1) $T$ has open values; and

(2) $T(A)=Y$.

Then there exists $B \in\langle A\rangle$ such that $F\left(\Gamma_{B}\right) \cap \bigcap\{T(b) \mid b \in B\} \neq \emptyset$.

Proof. Suppose that the conclusion does not hold. Define a map $G: A \multimap Y$ by $G(a):=Y \backslash T(a)$ for $a \in A$. Then each $G(a)$ is closed and, for each $B \in\langle A\rangle$,

$$
F\left(\Gamma_{B}\right) \subset \bigcup_{b \in B} Y \backslash T(b)=\bigcup_{b \in B} G(b)=G(B) .
$$

Since $F \in \mathfrak{K C}(X, Y)$, the family $\{G(a)\}_{a \in A}$ has the finite intersection property. Therefore, $\bigcap\{G(a) \mid a \in A\} \neq \emptyset$ and hence $T(A) \neq Y$, a contradiction.

REMARK. Note that Theorem $3.4^{\prime}$ generalizes a result of Balaj [1, Lemma 7], which is a particular case of Theorem $3.4^{\prime}$ for $F \in \mathfrak{A}_{c}^{\kappa}(X, Y)$, $X=D=Y$ and $F=1_{X}$.

The following is a new type of fixed point theorems:

Theorem 3.5. Let $(X, D ; \Gamma)$ be a $G$-convex space, $Y$ a topological space, $F \in \mathfrak{K O}(X, Y)$, and $A \in\langle D\rangle$. Let $G: A \multimap Y$ and $T: Y \multimap Y$ be two maps. Suppose that

(1) $F\left(\Gamma_{B}\right) \subset G(B)$ for each $B \in\langle A\rangle$;

(2) for each $y \in Y, T(y) \supset G(x)$ for some $x \in A$; and

(3) for each $z \in G(A), T^{-}(z)$ is closed.

Then $T$ has a fixed point.

Proof. Define $S: A \multimap Y$ by

$$
S(x):=\{y \in Y \mid G(x) \subset T(y)\} \text { for } x \in A .
$$

Then

$$
S(x)=\left\{y \in Y \mid y \in T^{-}(z) \text { for all } z \in G(x)\right\}=\bigcap_{z \in G(x)} T^{-}(z)
$$


and hence each $S(x)$ is closed by (3). Moreover, for each $y \in Y$, there is an $x \in A$ such that $G(x) \subset T(y)$ by (2), and hence $y \in S(x)$. This shows $Y=S(A)$. Therefore, by Theorem 3.4, there exist a $B \in\langle A\rangle$ and a $y_{0} \in Y$ such that $y_{0} \in F\left(\Gamma_{B}\right) \subset G(B)$ and $y_{0} \in S(b)$ for all $b \in B$. This implies $G(B) \subset T\left(y_{0}\right)$ and hence $y_{0} \in F\left(\Gamma_{B}\right) \subset G(B) \subset T\left(y_{0}\right)$.

ReankK. Note that Balaj [1, Theorem 2] is Theorem 3.5 for a $T_{1}$ regular space $Y$ and $F \in \mathfrak{A}_{c}^{\kappa}(X, Y)$. From Theorem 3.5 , we can also improve [1, Theorems 3. 4.6 and Corollary 5].

Finally, in this section, we give a simple proof of the following generalization of a coincidence theorem in Balaj [1, Theorem 8]:

Theoren 3.6. Let $(X \supset D ; \Gamma)$ be a $G$-convex space, $Z$ a nonempty set, and $F, T: X \multimap Z$ two maps such that

(1) for each $y \in X$, the set $\{x \in X \mid F(x) \cap T(y) \neq \emptyset\}$ is $\Gamma$-convex;

(2) for each $z \in F(X), T^{-}(z)$ is open; and

(3) $X=\bigcup_{x \in N}\{y \in X \mid F(x) \cap T(y) \neq \emptyset\}$ for some $N \in\langle D\rangle$.

Then there exists $x_{0} \in X$ such that $F\left(x_{0}\right) \cap T\left(x_{0}\right) \neq \emptyset$.

Proof. Define a map $G: X \multimap X$ by

$$
G(y):=\{x \in X \mid F(x) \cap T(y) \neq \emptyset\} \text { for } y \in X .
$$

Then each $G(y)$ is $\Gamma$-convex. On the other hand,

$$
\begin{aligned}
G^{-}(x) & =\{y \in X \mid F(x) \cap T(y) \neq \emptyset\} \\
& =\left\{y \in X \mid y \in T^{-}(z) \text { for some } z \in F(x)\right\} \\
& =\bigcup_{z \in F(x)} T^{-}(z)
\end{aligned}
$$

for $x \in X$. Then $G^{-}(x)$ is open as a union of open sets. By (3), $G^{-}(N)=X$ for some $N \in\langle D\rangle$. Therefore, by the Fan-Browder fixed point theorem for a $G$-convex spaces (Theorems 2.3 and $2.3^{\prime}$ ), $G$ has a fixed point $x_{0} \in X$; that is, $F\left(x_{0}\right) \cap T\left(x_{0}\right) \neq \emptyset$.

REMARK. The other results in [1, Theorems 9 and 10] are simple consequences of Theorem 3.6.

We note that our new results may have a large number of particular cases because of the abstract nature of the generalized convex space theory. and the readers could easily find such cases. 


\section{References}

[1] M. Balaj, Applications of two matching theorems in generalized convex spaces, Nonlinear Anal. Forum 7 (2002), 123130.

[2] S. Park. Foundations of the KKM theory 'ia coincidences of composites of upper semicontinuous maps, J. Korean Math. Soc. 31 (1994), 493-519.

[3] - Coincidence theorems for the better admissible multimaps and their applications, Nonlinear Anal. 30 (1997). 4183-4191.

[4] - A unified fixed point theory of multimaps on topological vector spaces, J. Korean Math. Soc. 35 (1998), 803 829. Corrections, ibid. 36 (1999), 829-832.

[5] - Elements of the KKM theory for generalized convex spaces, Korean $\mathbf{J}$. Comput. Appl. Math. 7 (2000), 1-28.

[6] - Remarks on topologies of generalized convex spaces, Nonlinear Funct. Anal. Appl. 5 (2000), 67.79.

[7] _ New topological versions of the Fan-Browder fixed point theorem, Nonlinear Anal. 47 (2001), 595606.

[8] - Fixed point theorems in locally G-convex spaces, Nonlinear Anal. 48 (2002), $869-879$.

[9] Coincidence, almost fixed point, and minimax theorems on generalized convex spaces, J. Nonlinear Convex Anal. 4 (2003), 151 164.

[10] S. Park and H. Kim, Coincidences of composites of u.s.c. maps on H-spaces and applications, J. Korean Math. Soc. 32 (1995), 251 264.

[11] Foundations of the KKM theory on generalized convex spaces, J. Math. Anal. Appl. 209 (1997), 551571.

[12] S. Park and W. Lee, A unified approach to generalized KKM maps in generalized convex spaces, J. Nonlinear Convex Anal. 2 (2001), 157166.

Hoonjoo Kim

Department of Mathematics Education

Daebul University

Young-Am 526-890, Korea

E-mail: hoonjoo@mail.daebul.ac.kr

Sehie Park

National Academy of Sciences

Seoul National University

Seoul 151-747, Korea

E-mail:shpark@math.snu.ac.kr 